\title{
Helmholtz Theorem for Nondifferentiable Hamiltonian Systems in the Framework of Cresson's Quantum Calculus
}

\author{
Frédéric Pierret $^{1}$ and Delfim F. M. Torres ${ }^{2}$ \\ ${ }^{1}$ Institut de Mécanique Céleste et de Calcul des Éphémérides, Observatoire de Paris, 75014 Paris, France \\ ${ }^{2}$ Center for Research and Development in Mathematics and Applications (CIDMA), Department of Mathematics, \\ University of Aveiro, 3810-193 Aveiro, Portugal \\ Correspondence should be addressed to Delfim F. M. Torres; delfim@ua.pt
}

Received 23 January 2016; Accepted 28 April 2016

Academic Editor: Taher S. Hassan

Copyright (C) 2016 F. Pierret and D. F. M. Torres. This is an open access article distributed under the Creative Commons Attribution License, which permits unrestricted use, distribution, and reproduction in any medium, provided the original work is properly cited.

We derive the Helmholtz theorem for nondifferentiable Hamiltonian systems in the framework of Cresson's quantum calculus. Precisely, we give a theorem characterizing nondifferentiable equations, admitting a Hamiltonian formulation. Moreover, in the affirmative case, we give the associated Hamiltonian.

\section{Introduction}

Several types of quantum calculus are available in the literature, including Jackson's quantum calculus $[1,2]$, Hahn's quantum calculus [3-5], the time-scale $q$-calculus $[6,7]$, the power quantum calculus [8], and the symmetric quantum calculus [9-11]. Cresson introduced in 2005 his quantum calculus on a set of Hölder functions [12]. This calculus attracted attention due to its applications in physics and the calculus of variations and has been further developed by several different authors (see [13-16] and references therein). Cresson's calculus of 2005 [12] presents, however, some difficulties, and in 2011 Cresson and Greff improved it $[17,18]$. Indeed, the quantum calculus of [12] let a free parameter, which is present in all the computations. Such parameter is certainly difficult to interpret. The new calculus of $[17,18]$ bypasses the problem by considering a quantity that is free of extra parameters and reduces to the classical derivative for differentiable functions. It is this new version of 2011 that we consider here, with a brief review of it being given in Section 2. Along the text, by Cresson's calculus we mean this quantum version of 2011 $[17,18]$. For the state of the art on the quantum calculus of variations we refer the reader to the recent book [19]. With respect to Cresson's approach, the quantum calculus of variations is still in its infancy: see [13,17, 18, 20-22]. In [17] nondifferentiable Euler-Lagrange equations are used in the study of PDEs. Euler-Lagrange equations for variational functionals with Lagrangians containing multiple quantum derivatives, depending on a parameter or containing higherorder quantum derivatives, are studied in [20]. Variational problems with constraints, with one and more than one independent variable, of first and higher-order type are investigated in [21]. Recently, problems of the calculus of variations and optimal control with time delay were considered [22]. In [18], a Noether type theorem is proved but only with the momentum term. This result is further extended in [23] by considering invariance transformations that also change the time variable, thus obtaining not only the generalized momentum term of [18] but also a new energy term. In [13], nondifferentiable variational problems with a free terminal point, with or without constraints, of first and higher-order are investigated. Here, we continue to develop Cresson's quantum calculus in obtaining a result for Hamiltonian systems and by considering the so-called inverse problem of the calculus of variations.

A classical problem in analysis is the well-known Helmholtz's inverse problem of the calculus of variations: find a necessary and sufficient condition under which a (system of) differential equation(s) can be written as an Euler-Lagrange or a Hamiltonian equation and, in the affirmative case, find all possible Lagrangian or Hamiltonian formulations. This condition is usually called the Helmholtz condition. The 
Lagrangian Helmholtz problem has been studied and solved by Douglas [24], Mayer [25], and Hirsch [26, 27]. The Hamiltonian Helmholtz problem has been studied and solved, up to our knowledge, by Santilli in his book [28]. Generalization of this problem in the discrete calculus of variations framework has been done in $[29,30]$, in the discrete Lagrangian case. In the case of time-scale calculus, that is, a mixing between continuous and discrete subintervals of time, see [31] for a necessary condition for a dynamic integrodifferential equation to be an Euler-Lagrange equation on time scales. For the Hamiltonian case it has been done for the discrete calculus of variations in [32] using the framework of [33] and in [34] using a discrete embedding procedure derived in [35]. In the case of time-scale calculus it has been done in [36]; for the Stratonovich stochastic calculus see [37]. Here we give the Helmholtz theorem for Hamiltonian systems in the case of nondifferentiable Hamiltonian systems in the framework of Cresson's quantum calculus. By definition, the nondifferentiable calculus extends the differentiable calculus. Such as in the discrete, time-scale, and stochastic cases, we recover the same conditions of existence of a Hamiltonian structure.

The paper is organized as follows. In Section 2, we give some generalities and notions about the nondifferentiable calculus introduced in [17], the so-called Cresson's quantum calculus. In Section 3, we remind definitions and results about classical and nondifferentiable Hamiltonian systems. In Section 4, we give a brief survey of the classical Helmholtz Hamiltonian problem and then we prove the main result of this paper-the nondifferentiable Hamiltonian Helmholtz theorem. Finally, we give two applications of our results in Section 5, and we end in Section 6 with conclusions and future work.

\section{Cresson's Quantum Calculus}

We briefly review the necessary concepts and results of the quantum calculus [17].

2.1. Definitions. Let $\mathbb{X}^{d}$ denote the set $\mathbb{R}^{d}$ or $\mathbb{C}^{d}, d \in \mathbb{N}$, and let $I$ be an open set in $\mathbb{R}$ with $[a, b] \subset I, a<b$. We denote by $\mathscr{F}\left(I, \mathbb{X}^{d}\right)$ the set of functions $f: I \rightarrow \mathbb{X}^{d}$ and by $\mathscr{C}^{0}\left(I, \mathbb{X}^{d}\right)$ the subset of functions of $\mathscr{F}\left(I, \mathbb{X}^{d}\right)$ which are continuous.

Definition 1 (Hölderian functions [17]). Let $f \in \mathscr{C}^{0}\left(I, \mathbb{R}^{d}\right)$. Let $t \in I$. Function $f$ is said to be $\alpha$-Hölderian, $0<\alpha<1$, at point $t$ if there exist positive constants $\epsilon>0$ and $c>0$ such that $\left|t-t^{\prime}\right| \leqslant \epsilon$ implies $\left\|f(t)-f\left(t^{\prime}\right)\right\| \leqslant c\left|t-t^{\prime}\right|^{\alpha}$ for all $t^{\prime} \in I$, where $\|\cdot\|$ is a norm on $\mathbb{R}^{d}$.

The set of Hölderian functions of Hölder exponent $\alpha$, for some $\alpha$, is denoted by $H^{\alpha}\left(I, \mathbb{R}^{d}\right)$. The quantum derivative is defined as follows.

Definition 2 (the $\epsilon$-left and $\epsilon$-right quantum derivatives [17]). Let $f \in \mathscr{C}^{0}\left(I, \mathbb{R}^{d}\right)$. For all $\epsilon>0$, the $\epsilon$-left and $\epsilon$-right quantum derivatives of $f$, denoted, respectively, by $d_{\epsilon}^{-} f$ and $d_{\epsilon}^{+} f$, are defined by

$$
\begin{aligned}
& d_{\epsilon}^{-} f(t)=\frac{f(t)-f(t-\epsilon)}{\epsilon}, \\
& d_{\epsilon}^{+} f(t)=\frac{f(t+\epsilon)-f(t)}{\epsilon} .
\end{aligned}
$$

Remark 3. The $\epsilon$-left and $\epsilon$-right quantum derivatives of a continuous function $f$ correspond to the classical derivative of the $\epsilon$-mean function $f_{\epsilon}^{\sigma}$ defined by

$$
f_{\epsilon}^{\sigma}(t)=\frac{\sigma}{\epsilon} \int_{t}^{t+\sigma \epsilon} f(s) d s, \quad \sigma= \pm .
$$

The next operator generalizes the classical derivative.

Definition 4 (the $\epsilon$-scale derivative [17]). Let $f \in \mathscr{C}^{0}\left(I, \mathbb{R}^{d}\right)$. For all $\epsilon>0$, the $\epsilon$-scale derivative of $f$, denoted by $\square_{\epsilon} f / \square t$, is defined by

$$
\frac{\square_{\epsilon} f}{\square t}=\frac{1}{2}\left[\left(d_{\epsilon}^{+} f+d_{\epsilon}^{-} f\right)+i \mu\left(d_{\epsilon}^{+} f-d_{\epsilon}^{-} f\right)\right],
$$

where $i$ is the imaginary unit and $\mu \in\{-1,1,0,-i, i\}$.

Remark 5. If $f$ is differentiable, then one can take the limit of the scale derivative when $\epsilon$ goes to zero. We then obtain the classical derivative $d f / d t$ of $f$.

We also need to extend the scale derivative to complex valued functions.

Definition 6 (see [17]). Let $f \in \mathscr{C}^{0}\left(I, \mathbb{C}^{d}\right)$ be a continuous complex valued function. For all $\epsilon>0$, the $\epsilon$-scale derivative of $f$, denoted by $\square_{\epsilon} f / \square t$, is defined by

$$
\frac{\square_{\epsilon} f}{\square t}=\frac{\square_{\epsilon} \operatorname{Re}(f)}{\square t}+i \frac{\square_{\epsilon} \operatorname{Im}(f)}{\square t},
$$

where $\operatorname{Re}(f)$ and $\operatorname{Im}(f)$ denote the real and imaginary part of $f$, respectively.

In Definition 4, the $\epsilon$-scale derivative depends on $\epsilon$, which is a free parameter related to the smoothing order of the function. This brings many difficulties in applications to physics, when one is interested in particular equations that do not depend on an extra parameter. To solve these problems, the authors of [17] introduced a procedure to extract information independent of $\epsilon$ but related with the mean behavior of the function.

Definition 7 (see [17]). Let $\left.\left.\mathscr{C}_{\text {conv }}^{0}(I \times] 0,1\right], \mathbb{R}^{d}\right) \subseteq \mathscr{C}^{0}(I \times] 0$, $\left.1], \mathbb{R}^{d}\right)$ be such that for any function $\left.\left.f \in \mathscr{C}_{\text {conv }}^{0}(I \times] 0,1\right], \mathbb{R}^{d}\right)$ the $\lim _{\epsilon \rightarrow 0} f(t, \epsilon)$ exists for any $t \in I$. We denote by $E$ a complementary space of $\left.\left.\mathscr{C}_{\text {conv }}^{0}(I \times] 0,1\right], \mathbb{R}^{d}\right)$ in $\left.\left.\mathscr{C}^{0}(I \times] 0,1\right], \mathbb{R}^{d}\right)$. We define the projection map $\pi$ by

$$
\begin{gathered}
\left.\left.\left.\left.\pi: \mathscr{C}_{\text {conv }}^{0}(I \times] 0,1\right], \mathbb{R}^{d}\right) \oplus E \longrightarrow \mathscr{C}_{\text {conv }}^{0}(I \times] 0,1\right], \mathbb{R}^{d}\right) \\
f_{\text {conv }}+f_{E} \longmapsto f_{\text {conv }}
\end{gathered}
$$


and the operator $\langle\cdot\rangle$ by

$$
\begin{aligned}
& \left.\left.\langle\cdot\rangle: \mathscr{C}^{0}(I \times] 0,1\right], \mathbb{R}^{d}\right) \longrightarrow \mathscr{C}^{0}\left(I, \mathbb{R}^{d}\right) \\
& f \longmapsto\langle f\rangle: t \longmapsto \lim _{\epsilon \rightarrow 0} \pi(f)(t, \epsilon) .
\end{aligned}
$$

The quantum derivative of $f$ without the dependence of $\epsilon$ is introduced in [17].

Definition 8 (see [17]). The quantum derivative of $f$ in the space $\mathscr{C}^{0}\left(I, \mathbb{R}^{d}\right)$ is given by

$$
\frac{\square f}{\square t}=\left\langle\frac{\square_{\epsilon} f}{\square t}\right\rangle .
$$

The quantum derivative (7) has some nice properties. Namely, it satisfies a Leibniz rule and a version of the fundamental theorem of calculus.

Theorem 9 (the quantum Leibniz rule [17]). Let $\alpha+\beta>1$. For $f \in H^{\alpha}\left(I, \mathbb{R}^{d}\right)$ and $g \in H^{\beta}\left(I, \mathbb{R}^{d}\right)$, one has

$$
\frac{\square}{\square t}(f \cdot g)(t)=\frac{\square f(t)}{\square t} \cdot g(t)+f(t) \cdot \frac{\square g(t)}{\square t} .
$$

Remark 10. For $f \in \mathscr{C}^{1}\left(I, \mathbb{R}^{d}\right)$ and $g \in \mathscr{C}^{1}\left(I, \mathbb{R}^{d}\right)$, one obtains from (8) the classical Leibniz rule: $(f \cdot g)^{\prime}=f^{\prime} \cdot g+$ $f \cdot g^{\prime}$.

Definition 11. We denote by $\mathscr{C}_{\square}^{1}$ the set of continuous functions $q \in \mathscr{C}^{0}\left([a, b], \mathbb{R}^{d}\right)$ such that $\square q / \square t \in \mathscr{C}^{0}\left(I, \mathbb{R}^{d}\right)$.

Theorem 12 (the quantum version of the fundamental theorem of calculus [17]). Let $f \in \mathscr{C}_{\square}^{1}\left([a, b], \mathbb{R}^{d}\right)$ be such that

$$
\lim _{\epsilon \rightarrow 0} \int_{a}^{b}\left(\frac{\square_{\epsilon} f}{\square t}\right)_{E}(t) d t=0 .
$$

Then,

$$
\int_{a}^{b} \frac{\square f}{\square t}(t) d t=f(b)-f(a) .
$$

2.2. Nondifferentiable Calculus of Variations. In [17] the calculus of variations with quantum derivatives is introduced and respective Euler-Lagrange equations derived without the dependence of $\epsilon$.

Definition 13. An admissible Lagrangian $L$ is a continuous function $L: \mathbb{R} \times \mathbb{R}^{d} \times \mathbb{C}^{d} \rightarrow \mathbb{C}$ such that $L(t, x, v)$ is holomorphic with respect to $v$ and differentiable with respect to $x$. Moreover, $L(t, x, v) \in \mathbb{R}$ when $v \in \mathbb{R}^{d} ; L(t, x, v) \in \mathbb{C}$ when $v \in \mathbb{C}^{d}$.

An admissible Lagrangian function $L: \mathbb{R} \times \mathbb{R}^{\mathrm{d}} \times \mathbb{C}^{d} \rightarrow \mathbb{C}$ defines a functional on $\mathscr{C}^{1}\left(I, \mathbb{R}^{d}\right)$, denoted by

$$
\begin{aligned}
\mathscr{L}: \mathscr{C}^{1}\left(I, \mathbb{R}^{d}\right) & \longrightarrow \mathbb{R} \\
q & \longmapsto \int_{a}^{b} L(t, q(t), \dot{q}(t)) d t .
\end{aligned}
$$

Extremals of the functional $\mathscr{L}$ can be characterized by the well-known Euler-Lagrange equation (see, e.g., [38]).

Theorem 14. The extremals $q \in \mathscr{C}^{1}\left(I, \mathbb{R}^{d}\right)$ of $\mathscr{L}$ coincide with the solutions of the Euler-Lagrange equation

$$
\frac{d}{d t}\left[\frac{\partial L}{\partial v}(t, q(t), \dot{q}(t))\right]=\frac{\partial L}{\partial x}(t, q(t), \dot{q}(t)) .
$$

The nondifferentiable embedding procedure allows us to define a natural extension of the classical Euler-Lagrange equation in the nondifferentiable context.

Definition 15 (see [17]). The nondifferentiable Lagrangian functional $\mathscr{L}_{\square}$ associated with $\mathscr{L}$ is given by

$$
\begin{aligned}
\mathscr{L}_{\square}: \mathscr{C}_{\square}^{1}\left(I, \mathbb{R}^{d}\right) & \longrightarrow \mathbb{R} \\
q & \longmapsto \int_{a}^{b} L\left(s, q(s), \frac{\square q(s)}{\square t}\right) d s .
\end{aligned}
$$

Let $H_{0}^{\beta}:=\left\{h \in H^{\beta}\left(I, \mathbb{R}^{d}\right), h(a)=h(b)=0\right\}$ and $q \epsilon$ $H^{\alpha}\left(I, \mathbb{R}^{d}\right)$ with $\alpha+\beta>1$. A $H_{0}^{\beta}$-variation of $q$ is a function of the form $q+h$, where $h \in H_{O}^{\beta}$. We denote by $D \mathscr{L}_{\square}(q)(h)$ the quantity

$$
\lim _{\epsilon \rightarrow 0} \frac{\mathscr{L}_{\square}(q+\epsilon h)-\mathscr{L}_{\square}(q)}{\epsilon}
$$

if there exists the so-called Fréchet derivative of $\mathscr{L}_{\square}$ at point $q$ in direction $h$.

Definition 16 (nondifferentiable extremals). A $H_{0}^{\beta}$-extremal curve of the functional $\mathscr{L}_{\square}$ is a curve $q \in H^{\alpha}\left(I, \mathbb{R}^{d}\right)$ satisfying $D \mathscr{L}_{\square}(q)(h)=0$ for any $h \in H_{0}^{\beta}$.

Theorem 17 (nondifferentiable Euler-Lagrange equations [17]). Let $0<\alpha, \beta<1$ with $\alpha+\beta>1$. Let $L$ be an admissible Lagrangian of class $\mathscr{C}^{2}$. We assume that $\gamma \in H^{\alpha}\left(I, \mathbb{R}^{d}\right)$, such that $\square \gamma / \square t \in H^{\alpha}\left(I, \mathbb{R}^{d}\right)$. Moreover, we assume that $L(t, \gamma(t)$, $\square \gamma(t) / \square t) h(t)$ satisfies condition (9) for all $h \in H_{0}^{\beta}\left(I, \mathbb{R}^{d}\right)$. A curve $\gamma$ satisfying the nondifferentiable Euler-Lagrange equation

$$
\frac{\square}{\square t}\left[\frac{\partial L}{\partial v}\left(t, \gamma(t), \frac{\square \gamma(t)}{\square t}\right)\right]=\frac{\partial L}{\partial x}\left(t, \gamma(t), \frac{\square \gamma(t)}{\square t}\right)
$$

is an extremal curve of functional (13).

\section{Reminder about Hamiltonian Systems}

We now recall the main concepts and results of both classical and Cresson's nondifferentiable Hamiltonian systems.

3.1. Classical Hamiltonian Systems. Let $L$ be an admissible Lagrangian function. If $L$ satisfies the so-called Legendre property, then we can associate to $L$ a Hamiltonian function denoted by $H$. 
Definition 18. Let $L$ be an admissible Lagrangian function. The Lagrangian $L$ is said to satisfy the Legendre property if the mapping $v \mapsto(\partial L / \partial v)(t, x, v)$ is invertible for any $(t, q, v) \epsilon$ $I \times \mathbb{R}^{d} \times \mathbb{C}^{d}$.

If we introduce a new variable

$$
p=\frac{\partial L}{\partial v}(t, q, v)
$$

and $L$ satisfies the Legendre property, then we can find a function $f$ such that

$$
v=f(t, q, p) .
$$

Using this notation, we have the following definition.

Definition 19. Let $L$ be an admissible Lagrangian function satisfying the Legendre property. The Hamiltonian function $H$ associated with $L$ is given by

$$
\begin{gathered}
H: \mathbb{R} \times \mathbb{R}^{d} \times \mathbb{C}^{d} \longrightarrow \mathbb{C} \\
(t, q, p) \longmapsto H(t, q, p)=p f(t, q, p)-L(t, q, f(t, q, p)) .
\end{gathered}
$$

We have the following theorem (see, e.g., [38]).

Theorem 20 (Hamilton's least-action principle). The curve $(q, p) \in \mathscr{C}\left(I, \mathbb{R}^{d}\right) \times \mathscr{C}\left(I, \mathbb{C}^{d}\right)$ is an extremal of the Hamiltonian functional

$$
\mathscr{H}(q, p)=\int_{a}^{b} p(t) \dot{q}(t)-H(t, q(t), p(t)) d t
$$

if and only if it satisfies the Hamiltonian system associated with $H$ given by

$$
\begin{aligned}
& \dot{q}(t)=\frac{\partial H(t, q(t), p(t))}{\partial p} \\
& \dot{p}(t)=-\frac{\partial H(t, q(t), p(t))}{\partial q}
\end{aligned}
$$

called the Hamiltonian equations.

A vectorial notation is obtained for the Hamiltonian equations in posing $z=(q, p)^{\top}$ and $\nabla H=(\partial H / \partial q, \partial H / \partial p)^{\top}$, where $T$ denotes the transposition. The Hamiltonian equations are then written as

$$
\frac{d z(t)}{d t}=J \cdot \nabla H(t, z(t))
$$

where

$$
J=\left(\begin{array}{cc}
0 & I_{d} \\
-I_{d} & 0
\end{array}\right)
$$

denotes the symplectic matrix with $I_{d}$ being the identity matrix on $\mathbb{R}^{d}$.
3.2. Nondifferentiable Hamiltonian Systems. The nondifferentiable embedding induces a change in the phase space with respect to the classical case. As a consequence, we have to work with variables $(x, p)$ that belong to $\mathbb{R}^{d} \times \mathbb{C}^{d}$ and not only to $\mathbb{R}^{d} \times \mathbb{R}^{d}$, as usual.

Definition 21 (nondifferentiable embedding of Hamiltonian systems [17]). The nondifferentiable embedded Hamiltonian system (20) is given by

$$
\begin{aligned}
& \frac{\square q(t)}{\square t}=\frac{\partial H(t, q(t), p(t))}{\partial p}, \\
& \frac{\square p(t)}{\square t}=-\frac{\partial H(t, q(t), p(t))}{\partial q}
\end{aligned}
$$

and the embedded Hamiltonian functional $\mathscr{H}_{\square}$ is defined on $H^{\alpha}\left(I, \mathbb{R}^{d}\right) \times H^{\alpha}\left(I, \mathbb{C}^{d}\right)$ by

$$
\mathscr{H}_{\square}(q, p)=\int_{a}^{b}\left(p(t) \frac{\square q(t)}{\square t}-H(t, q(t), p(t))\right) d t .
$$

The nondifferentiable calculus of variations allows us to derive the extremals for $\mathscr{H}_{\square}$.

Theorem 22 (nondifferentiable Hamilton's least-action principle [17]). Let $0<\alpha, \beta<1$ with $\alpha+\beta>1$. Let $L$ be an admissible $\mathscr{C}^{2}$-Lagrangian. We assume that $\gamma \in H^{\alpha}\left(I, \mathbb{R}^{d}\right)$, such that $\square \gamma / \square t \in H^{\alpha}\left(I, \mathbb{R}^{d}\right)$. Moreover, we assume that $L(t, \gamma(t), \square \gamma(t) / \square t) h(t)$ satisfies condition (9) for all $h \in$ $H_{0}^{\beta}\left(I, \mathbb{R}^{d}\right)$. Let $H$ be the corresponding Hamiltonian defined by (18). A curve $\gamma \mapsto(t, q(t), p(t)) \in I \times \mathbb{R}^{d} \times \mathbb{C}^{d}$ solution of the nondifferentiable Hamiltonian system (23) is an extremal of functional (24) over the space of variations $V=H_{0}^{\beta}\left(I, \mathbb{R}^{d}\right) \times$ $H_{0}^{\beta}\left(I, \mathbb{C}^{d}\right)$.

\section{Nondifferentiable Helmholtz Problem}

In this section, we solve the inverse problem of the nondifferentiable calculus of variations in the Hamiltonian case. We first recall the usual way to derive the Helmholtz conditions following the presentation made by Santilli [28]. Two main derivations are available:

(i) The first is related to the characterization of Hamiltonian systems via the symplectic two-differential form and the fact that by duality the associated onedifferential form to a Hamiltonian vector field is closed-the so-called integrability conditions.

(ii) The second uses the characterization of Hamiltonian systems via the self-adjointness of the Fréchet derivative of the differential operator associated with the equation-the so-called Helmholtz conditions.

Of course, we have coincidence of the two procedures in the classical case. As there is no analogous of differential form in the framework of Cresson's quantum calculus, we follow the second way to obtain the nondifferentiable analogue of 
the Helmholtz conditions. For simplicity, we consider a timeindependent Hamiltonian. The time-dependent case can be done in the same way.

4.1. Helmholtz Conditions for Classical Hamiltonian Systems. In this section we work on $\mathbb{R}^{2 d}, d \geq 1, d \in \mathbb{N}$.

4.1.1. Symplectic Scalar Product. The symplectic scalar product $\langle\cdot, \cdot\rangle_{J}$ is defined by

$$
\langle X, Y\rangle_{J}=\langle X, J \cdot Y\rangle
$$

for all $X, Y \in \mathbb{R}^{2 d}$, where $\langle\cdot, \cdot\rangle$ denotes the usual scalar product and $J$ is the symplectic matrix (22). We also consider the $L^{2}$ symplectic scalar product induced by $\langle\cdot, \cdot\rangle_{J}$ defined for $f, g \in \mathscr{C}^{0}\left([a, b], \mathbb{R}^{2 d}\right)$ by

$$
\langle f, g\rangle_{L^{2}, J}=\int_{a}^{b}\langle f(t), g(t)\rangle_{J} d t
$$

4.1.2. Adjoint of a Differential Operator. In the following, we consider first-order differential equations of the form

$$
\frac{d}{d t}\left(\begin{array}{l}
q \\
p
\end{array}\right)=\left(\begin{array}{l}
X_{q}(q, p) \\
X_{p}(q, p)
\end{array}\right)
$$

where the vector fields $X_{q}$ and $X_{p}$ are $\mathscr{C}^{1}$ with respect to $q$ and $p$. The associated differential operator is written as

$$
O_{X}(q, p)=\left(\begin{array}{l}
\dot{q}-X_{q}(q, p) \\
\dot{p}-X_{p}(q, p)
\end{array}\right)
$$

A natural notion of adjoint for a differential operator is then defined as follows.

Definition 23. Let $A: \mathscr{C}^{1}\left([a, b], \mathbb{R}^{2 d}\right) \rightarrow \mathscr{C}^{1}\left([a, b], \mathbb{R}^{2 d}\right)$. We define the adjoint $A_{J}^{*}$ of $A$ with respect to $\langle\cdot, \cdot\rangle_{L^{2}, J}$ by

$$
\langle A \cdot f, g\rangle_{L^{2}, J}=\left\langle A_{J}^{*} \cdot g, f\right\rangle_{L^{2}, J}
$$

An operator $A$ will be called self-adjoint if $A=A_{J}^{*}$ with respect to the $L^{2}$ symplectic scalar product.

4.1.3. Hamiltonian Helmholtz Conditions. The Helmholtz conditions in the Hamiltonian case are given by the following result (see Theorem 3.12.1, p. 176-177 in [28]).

Theorem 24 (Hamiltonian Helmholtz theorem). Let $X(q, p)$ be a vector field defined by $X(q, p)^{\top}=\left(X_{q}(q, p), X_{p}(q, p)\right)$. The differential equation (27) is Hamiltonian if and only if the associated differential operator $\mathrm{O}_{X}$ given by (28) has a selfadjoint Fréchet derivative with respect to the $L^{2}$ symplectic scalar product. In this case the Hamiltonian is given by

$$
H(q, p)=\int_{0}^{1}\left[p \cdot X_{q}(\lambda q, \lambda p)-q \cdot X_{p}(\lambda q, \lambda p)\right] d \lambda
$$

The conditions for the self-adjointness of the differential operator can be made explicit. They coincide with the integrability conditions characterizing the exactness of the one-form associated with the vector field by duality (see [28], Theorem 2.7.3, p. 88).

Theorem 25 (integrability conditions). Let $X(q, p)^{\top}=$ $\left(X_{q}(q, p), X_{p}(q, p)\right)$ be a vector field. The differential operator $\mathrm{O}_{X}$ given by (28) has a self-adjoint Fréchet derivative with respect to the $L^{2}$ symplectic scalar product if and only if

$$
\frac{\partial X_{q}}{\partial q}+\left(\frac{\partial X_{p}}{\partial p}\right)^{\top}=0, \quad \frac{\partial X_{q}}{\partial p}, \frac{\partial X_{p}}{\partial q} \text { are symmetric. }
$$

4.2. Helmholtz Conditions for Nondifferentiable Hamiltonian Systems. The previous scalar products extend naturally to complex valued functions. Let $0<\alpha<1$ and let $(q, p) \in$ $H^{\alpha}\left(I, \mathbb{R}^{d}\right) \times H^{\alpha}\left(I, \mathbb{C}^{d}\right)$, such that $\square q / \square t \in H^{\alpha}\left(I, \mathbb{C}^{d}\right)$ and $\square p / \square t \in H^{\alpha}\left(I, \mathbb{C}^{d}\right)$. We consider first-order nondifferential equations of the form

$$
\frac{\square}{\square t}\left(\begin{array}{l}
q \\
p
\end{array}\right)=\left(\begin{array}{l}
X_{q}(q, p) \\
X_{p}(q, p)
\end{array}\right) .
$$

The associated quantum differential operator is written as

$$
O_{\square, X}(q, p)=\left(\begin{array}{l}
\frac{\square q}{\square t}-X_{q}(q, p) \\
\frac{\square p}{\square t}-X_{p}(q, p)
\end{array}\right) .
$$

A natural notion of adjoint for a quantum differential operator is then defined.

Definition 26. Let $A: \mathscr{C}_{\square}^{1}\left([a, b], \mathbb{C}^{2 d}\right) \rightarrow \mathscr{C}_{\square}^{1}\left([a, b], \mathbb{C}^{2 d}\right)$. We define the adjoint $A_{J}^{*}$ of $A$ with respect to $\langle\cdot, \cdot\rangle_{L^{2}, J}$ by

$$
\langle A \cdot f, g\rangle_{L^{2}, J}=\left\langle A_{J}^{*} \cdot g, f\right\rangle_{L^{2}, J}
$$

An operator $A$ will be called self-adjoint if $A=A_{J}^{*}$ with respect to the $L^{2}$ symplectic scalar product. We can now obtain the adjoint operator associated with $O_{\square, X}$

Proposition 27. Let $\beta$ be such that $\alpha+\beta>1$. Let $(u, v) \in$ $H_{0}^{\beta}\left(I, \mathbb{R}^{d}\right) \times H_{0}^{\beta}\left(I, \mathbb{C}^{d}\right)$, such that $\square u / \square t \in H^{\alpha}\left(I, \mathbb{C}^{d}\right)$ and $\square v / \square t \in H^{\alpha}\left(I, \mathbb{C}^{d}\right)$. The Fréchet derivative $D O_{\square, X}$ of (33) at $(q, p)$ along $(u, v)$ is then given by

$$
D O_{\square, X}(q, p)(u, v)=\left(\begin{array}{l}
\frac{\square u}{\square t}-\frac{\partial X_{q}}{\partial q} \cdot u-\frac{\partial X_{q}}{\partial p} \cdot v \\
\frac{\square v}{\square t}-\frac{\partial X_{p}}{\partial q} \cdot u-\frac{\partial X_{p}}{\partial p} \cdot v
\end{array}\right) .
$$


Assume that $u \cdot h$ and $v \cdot h$ satisfy condition (9) for any $h \in$ $H_{0}^{\beta}\left(I, \mathbb{C}^{d}\right)$. In consequence, the adjoint $D O_{\square, X}^{*}$ of $D O_{\square, X}(q, p)$ with respect to the $L^{2}$ symplectic scalar product is given by

$$
\begin{aligned}
D O_{\square, X}^{*}(q, p)(u, v) & \left(\begin{array}{l}
\frac{\square u}{\square t}+\left(\frac{\partial X_{p}}{\partial p}\right)^{\top} \cdot u-\left(\frac{\partial X_{q}}{\partial p}\right)^{\top} \cdot v \\
\frac{\square v}{\square t}-\left(\frac{\partial X_{p}}{\partial q}\right)^{\top} \cdot u+\left(\frac{\partial X_{q}}{\partial q}\right)^{\top} \cdot v
\end{array}\right) .
\end{aligned}
$$

Proof. The expression for the Fréchet derivative of (33) at $(q, p)$ along $(u, v)$ is a simple computation. Let $(w, x) \in$ $H_{0}^{\beta}\left(I, \mathbb{R}^{d}\right) \times H_{0}^{\beta}\left(I, \mathbb{C}^{d}\right)$ be such that $\square w / \square t \in H^{\alpha}\left(I, \mathbb{C}^{d}\right)$ and $\square x / \square t \in H^{\alpha}\left(I, \mathbb{C}^{d}\right)$. By definition, we have

$$
\begin{aligned}
\left\langle D O_{\square, X}(q, p)(u, v),(w, x)\right\rangle_{L^{2}, J} & =\int_{a}^{b}\left[\frac{\square u}{\square t} \cdot x\right. \\
& -\left(\frac{\partial X_{q}}{\partial q} \cdot u\right) \cdot x-\left(\frac{\partial X_{q}}{\partial p} \cdot v\right) \cdot x-\frac{\square v}{\square t} \cdot w \\
& \left.+\left(\frac{\partial X_{p}}{\partial q} \cdot u\right) \cdot w+\left(\frac{\partial X_{p}}{\partial p} \cdot v\right) \cdot w\right] d t .
\end{aligned}
$$

As $u \cdot h$ and $v \cdot h$ satisfy condition (9) for any $h \in H_{0}^{\beta}\left(I, \mathbb{C}^{d}\right)$, using the quantum Leibniz rule and the quantum version of the fundamental theorem of calculus, we obtain

$$
\begin{aligned}
& \int_{a}^{b} \frac{\square u}{\square t} \cdot b d t=\int_{a}^{b}-u \cdot \frac{\square b}{\square t} d t, \\
& \int_{a}^{b} \frac{\square v}{\square t} \cdot a d t=\int_{a}^{b}-u \cdot \frac{\square b}{\square t} d t .
\end{aligned}
$$

Then,

$$
\begin{gathered}
\left\langle D O_{\square, X}(q, p)(u, v),(w, x)\right\rangle_{L^{2}, J}=\int_{a}^{b}[-u \\
\cdot\left(\frac{\square x}{\square t}-\left(\frac{\partial X_{p}}{\partial q}\right)^{\top} \cdot w+\left(\frac{\partial X_{q}}{\partial q}\right)^{\top} \cdot x\right)+v \\
\left.\cdot\left(\frac{\square w}{\square t}+\left(\frac{\partial X_{p}}{\partial p}\right)^{\top} \cdot w-\left(\frac{\partial X_{q}}{\partial p}\right)^{\top} \cdot x\right)\right] d t .
\end{gathered}
$$

By definition, we obtain the expression of the adjoint $D O_{\square, X}^{*}$ of $D O_{\square, X}(q, p)$ with respect to the $L^{2}$ symplectic scalar product.

In consequence, from a direct identification, we obtain the nondifferentiable self-adjointess conditions called Helmholtz's conditions. As in the classical case, we call these conditions nondifferentiable integrability conditions.

Theorem 28 (nondifferentiable integrability conditions). Let $X(q, p)^{\top}=\left(X_{q}(q, p), X_{p}(q, p)\right)$ be a vector field. The differential operator $O_{\square, X}$ given by (33) has a self-adjoint Fréchet derivative with respect to the symplectic scalar product if and only if

$$
\begin{gathered}
\frac{\partial X_{q}}{\partial q}+\left(\frac{\partial X_{p}}{\partial p}\right)^{\top}=0, \\
\frac{\partial X_{q}}{\partial p}, \frac{\partial X_{p}}{\partial q} \text { are symmetric. }
\end{gathered}
$$

Remark 29. One can see that the Helmholtz conditions are the same as in the classical, discrete, time-scale, and stochastic cases. We expected such a result because Cresson's quantum calculus provides a quantum Leibniz rule and a quantum version of the fundamental theorem of calculus. If such properties of an underlying calculus exist, then the Helmholtz conditions will always be the same up to some conditions on the working space of functions.

We now obtain the main result of this paper, which is the Helmholtz theorem for nondifferentiable Hamiltonian systems.

Theorem 30 (nondifferentiable Hamiltonian Helmholtz theorem). Let $X(q, p)$ be a vector field defined by $X(q, p)^{\top}=$ $\left(X_{q}(q, p), X_{p}(q, p)\right)$. The nondifferentiable system of (32) is Hamiltonian if and only if the associated quantum differential operator $\mathrm{O}_{\square, X}$ given by (33) has a self-adjoint Fréchet derivative with respect to the $L^{2}$ symplectic scalar product. In this case, the Hamiltonian is given by

$$
H(q, p)=\int_{0}^{1}\left[p \cdot X_{q}(\lambda q, \lambda p)-q \cdot X_{p}(\lambda q, \lambda p)\right] d \lambda .
$$

Proof. If $X$ is Hamiltonian, then there exists a function $H$ : $\mathbb{R}^{d} \times \mathbb{C}^{d} \rightarrow \mathbb{C}$ such that $H(q, p)$ is holomorphic with respect to $v$ and differentiable with respect to $q$ and $X_{q}=\partial H / \partial p$ and $X_{p}=-\partial H / \partial q$. The nondifferentiable integrability conditions are clearly verified using Schwarz's lemma. Reciprocally, we assume that $X$ satisfies the nondifferentiable integrability conditions. We will show that $X$ is Hamiltonian with respect to the Hamiltonian

$$
H(q, p)=\int_{0}^{1}\left[p \cdot X_{q}(\lambda q, \lambda p)-q \cdot X_{p}(\lambda q, \lambda p)\right] d \lambda ;
$$

that is, we must show that

$$
\begin{aligned}
& X_{q}(q, p)=\frac{\partial H(q, p)}{\partial p}, \\
& X_{p}(q, p)=-\frac{\partial H(q, p)}{\partial q} .
\end{aligned}
$$

We have

$$
\begin{gathered}
\frac{\partial H(q, p)}{\partial q}=\int_{0}^{1}\left[p \cdot \lambda \frac{\partial X_{q}(\lambda q, \lambda p)}{\partial q}-X_{p}(\lambda q, \lambda p)\right. \\
\left.-q \cdot \lambda \frac{\partial X_{p}(\lambda q, \lambda p)}{\partial q}\right] d \lambda,
\end{gathered}
$$




$$
\begin{aligned}
& \frac{\partial H(q, p)}{\partial p}=\int_{0}^{1}\left[X_{p}(\lambda q, \lambda p)+p \cdot \lambda \frac{\partial X_{q}(\lambda q, \lambda p)}{\partial p}\right. \\
& \left.-q \cdot \lambda \frac{\partial X_{p}(\lambda q, \lambda p)}{\partial p}\right] d \lambda .
\end{aligned}
$$

Using the nondifferentiable integrability conditions, we obtain

$$
\begin{aligned}
& \frac{\partial H(q, p)}{\partial q}=\int_{0}^{1}-\frac{\partial\left(\lambda X_{p}(\lambda q, \lambda p)\right)}{\partial \lambda} d \lambda=-X_{p}(q, p), \\
& \frac{\partial H(q, p)}{\partial q}=\int_{0}^{1} \frac{\partial\left(\lambda X_{q}(\lambda q, \lambda p)\right)}{\partial \lambda} d \lambda=X_{q}(q, p),
\end{aligned}
$$

which concludes the proof.

\section{Applications}

We now provide two illustrative examples of our results: one with the formulation of dynamical systems with linear parts and another with Newton's equation, which is particularly useful to study partial differentiable equations such as the Navier-Stokes equation. Indeed, the Navier-Stokes equation can be recovered from a Lagrangian structure with Cresson's quantum calculus [17]. For more applications see [34].

Let $0<\alpha<1$ and let $(q, p) \in H^{\alpha}\left(I, \mathbb{R}^{d}\right) \times H^{\alpha}\left(I, \mathbb{C}^{d}\right)$ be such that $\square q / \square t \in H^{\alpha}\left(I, \mathbb{C}^{d}\right)$ and $\square q / \square t \in H^{\alpha}\left(I, \mathbb{C}^{d}\right)$.

5.1. The Linear Case. Let us consider the discrete nondifferentiable system

$$
\begin{aligned}
& \frac{\square q}{\square t}=\alpha q+\beta p, \\
& \frac{\square p}{\square t}=\gamma q+\delta p,
\end{aligned}
$$

where $\alpha, \beta, \gamma$, and $\delta$ are constants. The Helmholtz condition (HC2) is clearly satisfied. However, system (45) satisfies the condition ( $\mathrm{HC} 1$ ) if and only if $\alpha+\delta=0$. As a consequence, linear Hamiltonian nondifferentiable equations are of the form

$$
\begin{aligned}
& \frac{\square q}{\square t}=\alpha q+\beta p, \\
& \frac{\square p}{\square t}=\gamma q-\alpha p .
\end{aligned}
$$

Using formula (40), we compute explicitly the Hamiltonian, which is given by

$$
H(q, p)=\frac{1}{2}\left(\beta p^{2}-\gamma q^{2}\right)+\alpha q \cdot p .
$$

5.2. Newton's Equation. Newton's equation (see [38]) is given by

$$
\begin{aligned}
& \dot{q}=\frac{p}{m}, \\
& \dot{p}=-U^{\prime}(q),
\end{aligned}
$$

with $m \in \mathbb{R}^{+}$and $q, p \in \mathbb{R}^{d}$. This equation possesses a natural Hamiltonian structure with the Hamiltonian given by

$$
H(q, p)=\frac{1}{2 m} p^{2}+U(q) \text {. }
$$

Using Cresson's quantum calculus, we obtain a natural nondifferentiable system given by

$$
\begin{aligned}
& \frac{\square q}{\square t}=\frac{p}{m}, \\
& \frac{\square p}{\square t}=-U^{\prime}(q) .
\end{aligned}
$$

The Hamiltonian Helmholtz conditions are clearly satisfied.

Remark 31. It must be noted that Hamiltonian (49) associated with (50) is recovered by formula (40).

\section{Conclusion}

We proved a Helmholtz theorem for nondifferentiable equations, which gives necessary and sufficient conditions for the existence of a Hamiltonian structure. In the affirmative case, the Hamiltonian is given. Our result extends the results of the classical case when restricting attention to differentiable functions. An important complementary result for the nondifferentiable case is to obtain the Helmholtz theorem in the Lagrangian case. This is nontrivial and will be subject of future research.

\section{Competing Interests}

The authors declare that they have no competing interests.

\section{Acknowledgments}

This work was supported by FCT and CIDMA through project UID/MAT/04106/2013. The first author is grateful to CIDMA and DMat-UA for the hospitality and good working conditions during his visit at University of Aveiro. The authors would like to thank an anonymous referee for careful reading of the submitted paper and for useful suggestions.

\section{References}

[1] V. Kac and P. Cheung, Quantum Calculus, Universitext, Springer, New York, NY, USA, 2002.

[2] N. Martins and D. F. M. Torres, "Higher-order infinite horizon variational problems in discrete quantum calculus," Computers \& Mathematics with Applications, vol. 64, no. 7, pp. 2166-2175, 2012.

[3] A. M. C. Brito da Cruz, N. Martins, and D. F. M. Torres, "Higherorder Hahn's quantum variational calculus," Nonlinear Analysis: Theory, Methods \& Applications, vol. 75, no. 3, pp. 1147-1157, 2012.

[4] A. B. Malinowska and N. Martins, "Generalized transversality conditions for the Hahn quantum variational calculus," Optimization, vol. 62, no. 3, pp. 323-344, 2013. 
[5] A. B. Malinowska and D. F. M. Torres, "The Hahn quantum variational calculus," Journal of Optimization Theory and Applications, vol. 147, no. 3, pp. 419-442, 2010.

[6] M. Bohner and A. Peterson, Dynamic Equations on Time Scales. An Introduction with Applications, Birkhäauser, Boston, Mass, USA, 2001.

[7] N. Martins and D. F. M. Torres, "L'Hôpital-type rules for monotonicity with application to quantum calculus," International Journal of Mathematics and Computation, vol. 10, no. M11, pp. 99-106, 2011.

[8] K. A. Aldwoah, A. B. Malinowska, and D. F. M. Torres, "The power quantum calculus and variational problems," Dynamics of Continuous, Discrete \& Impulsive Systems Series B: Applications \& Algorithms, vol. 19, no. 1-2, pp. 93-116, 2012.

[9] A. M. C. Brito da Cruz, N. Martins, and D. F. M. Torres, "Hahn's symmetric quantum variational calculus," Numerical Algebra, Control and Optimization, vol. 3, no. 1, pp. 77-94, 2013.

[10] A. M. C. Brito da Cruz, N. Martins, and D. F. M. Torres, "A symmetric Nörlund sum with application to inequalities," in Differential and Difference Equations with Applications, S. Pinelas, M. Chipot, and Z. Dosla, Eds., vol. 47 of Springer Proceedings in Mathematics \& Statistics, pp. 495-503, Springer, New York, NY, USA, 2013.

[11] A. M. C. Brito da Cruz, N. Martins, and D. F. M. Torres, "A symmetric quantum calculus," in Differential and Difference Equations with Applications, vol. 47 of Springer Proceedings in Mathematics \& Statistics, pp. 359-366, Springer, New York, NY, USA, 2013.

[12] J. Cresson, "Non-differentiable variational principles," Journal of Mathematical Analysis and Applications, vol. 307, no. 1, pp. 48-64, 2005.

[13] R. Almeida and N. Martins, "Variational problems for Hölderian functions with free terminal point," Mathematical Methods in the Applied Sciences, vol. 38, no. 6, pp. 1059-1069, 2015.

[14] R. Almeida and D. F. M. Torres, "Hölderian variational problems subject to integral constraints," Journal of Mathematical Analysis and Applications, vol. 359, no. 2, pp. 674-681, 2009.

[15] C. Castro, "On nonlinear quantum mechanics, noncommutative phase spaces, fractal-scale calculus and vacuum energy," Foundations of Physics, vol. 40, no. 11, pp. 1712-1730, 2010.

[16] J. Cresson, G. S. F. Frederico, and D. F. M. Torres, "Constants of motion for non-differentiable quantum variational problems," Topological Methods in Nonlinear Analysis, vol. 33, no. 2, pp. 217231, 2009.

[17] J. Cresson and I. Greff, "Non-differentiable embedding of Lagrangian systems and partial differential equations," Journal of Mathematical Analysis and Applications, vol. 384, no. 2, pp. 626-646, 2011.

[18] J. Cresson and I. Greff, "A non-differentiable Noether's theorem," Journal of Mathematical Physics, vol. 52, no. 2, Article ID 023513, 10 pages, 2011.

[19] A. B. Malinowska and D. F. M. Torres, Quantum Variational Calculus, Springer Briefs in Control, Automation and Robotics, Springer, New York, NY, USA, 2014.

[20] R. Almeida and D. F. M. Torres, "Generalized Euler-Lagrange equations for variational problems with scale derivatives," Letters in Mathematical Physics, vol. 92, no. 3, pp. 221-229, 2010.

[21] R. Almeida and D. F. M. Torres, "Nondifferentiable variational principles in terms of a quantum operator," Mathematical Methods in the Applied Sciences, vol. 34, no. 18, pp. 2231-2241, 2011.
[22] G. S. F. Frederico and D. F. M. Torres, "A nondifferentiable quantum variational embedding in presence of time delays," International Journal of Difference Equations, vol. 8, no. 1, pp. 49-62, 2013.

[23] G. S. F. Frederico and D. F. M. Torres, "Noether's theorem with momentum and energy terms for Cresson's quantum variational problems," Advances in Dynamical Systems and Applications, vol. 9, no. 2, pp. 179-189, 2014.

[24] J. Douglas, "Solution of the inverse problem of the calculus of variations," Transactions of the American Mathematical Society, vol. 50, pp. 71-128, 1941.

[25] A. Mayer, "Die existenzbedingungen eines kinetischen potentiales," Bericht Verhand. König. Sächs. Gesell. Wiss.Leipzig, Math.-Phys. Klasse, vol. 84, pp. 519-529, 1896.

[26] A. Hirsch, "Ueber eine charakteristische Eigenschaft der Differentialgleichungen der Variationsrechnung," Mathematische Annalen, vol. 49, no. 1, pp. 49-72, 1897.

[27] A. Hirsch, "Die existenzbedingungen des verallgemeinerten kinetischen potentials," Mathematische Annalen, vol. 50, no. 2, pp. 429-441, 1898.

[28] R. Santilli, Foundations of Theoretical Mechanics: The Inverse Problem in Newtonian Mechanics, Texts and Monographs in Physics, Springer, New York, NY, USA, 1978.

[29] L. Bourdin and J. Cresson, "Helmholtz's inverse problem of the discrete calculus of variations," Journal of Difference Equations and Applications, vol. 19, no. 9, pp. 1417-1436, 2013.

[30] P. E. Hydon and E. L. Mansfield, "A variational complex for difference equations," Foundations of Computational Mathematics, vol. 4, no. 2, pp. 187-217, 2004.

[31] M. Dryl and D. F. M. Torres, "Necessary condition for an Euler-Lagrange equation on time scales," Abstract and Applied Analysis, vol. 2014, Article ID 631281, 7 pages, 2014.

[32] I. D. Albu and D. Opris, "Helmholtz type condition for mechanical integrators," Novi Sad Journal of Mathematics, vol. 29, no. 3, pp. 11-21, 1999.

[33] J. E. Marsden and M. West, "Discrete mechanics and variational integrators," Acta Numerica, vol. 10, pp. 357-514, 2001.

[34] J. Cresson and F. Pierret, "Continuous versusdiscrete structures II-discrete Hamiltonian systems and Helmholtz conditions," http://arxiv.org/abs/1501.03203.

[35] J. Cresson and F. Pierret, "Continuous versus discretestructures I-discrete embeddings and ordinary differential equations," http://arxiv.org/abs/1411.7117.

[36] F. Pierret, "Helmholtz theorem for Hamiltonian systems on time scales," International Journal of Difference Equations, vol. 10, no. 1, pp. 121-135, 2015.

[37] F. Pierret, "Helmholtz theorem for stochastic Hamiltonian systems," Advances in Dynamical Systems and Applications, vol. 10, no. 2, pp. 201-214, 2015.

[38] V. I. Arnold, Mathematical Methods of Classical Mechanics, Graduate Texts in Mathematics, Springer, New York, NY, USA, 1978. 


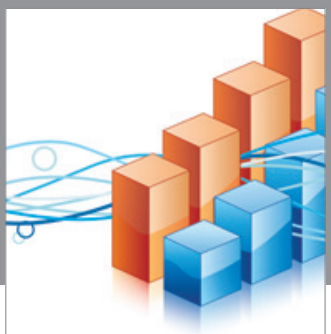

Advances in

Operations Research

vatem alat4

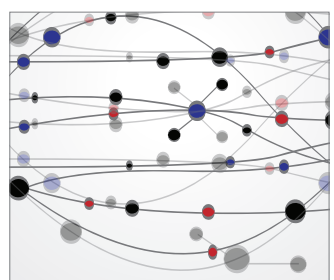

\section{The Scientific} World Journal
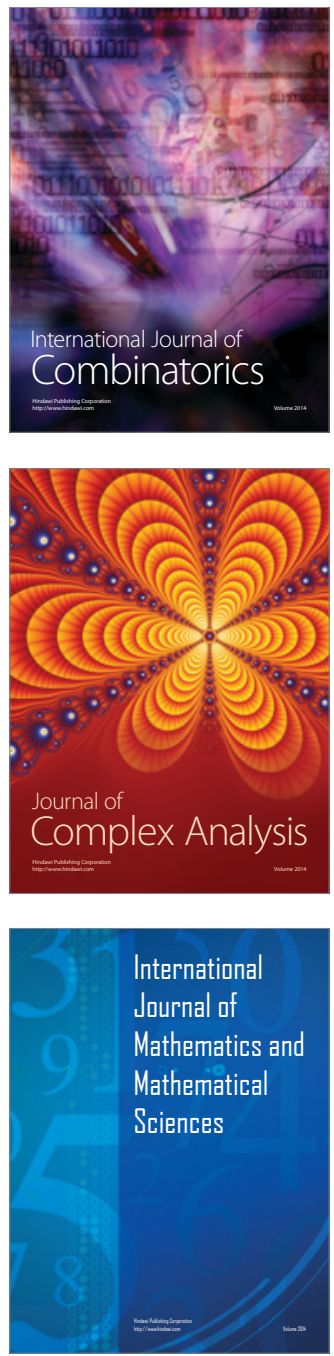
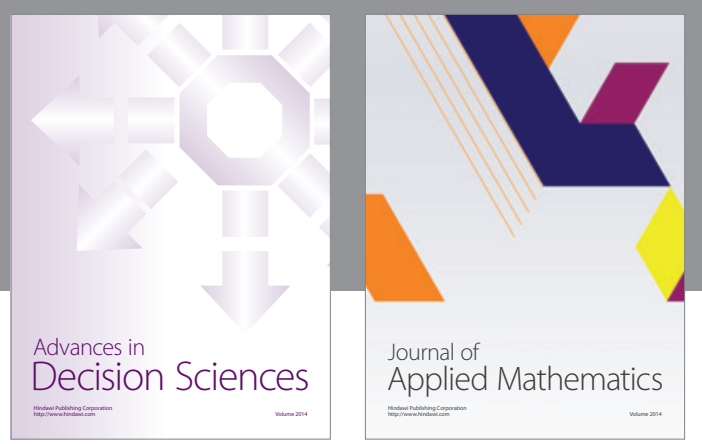

Algebra



\section{Hindawi}

Submit your manuscripts at

http://www.hindawi.com
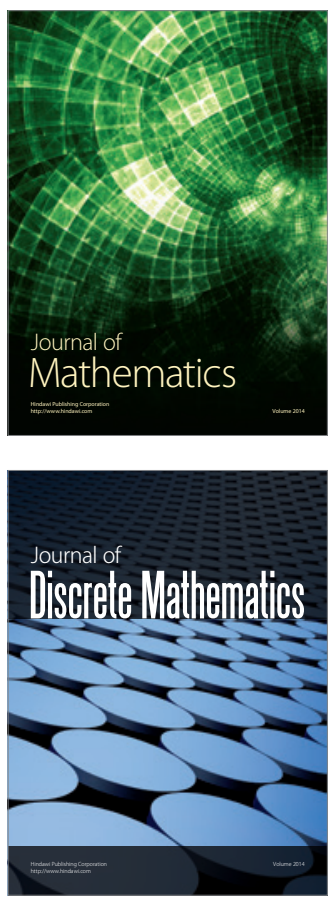

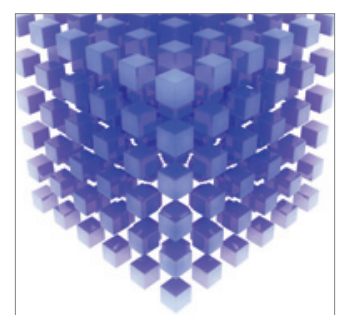

Mathematical Problems in Engineering
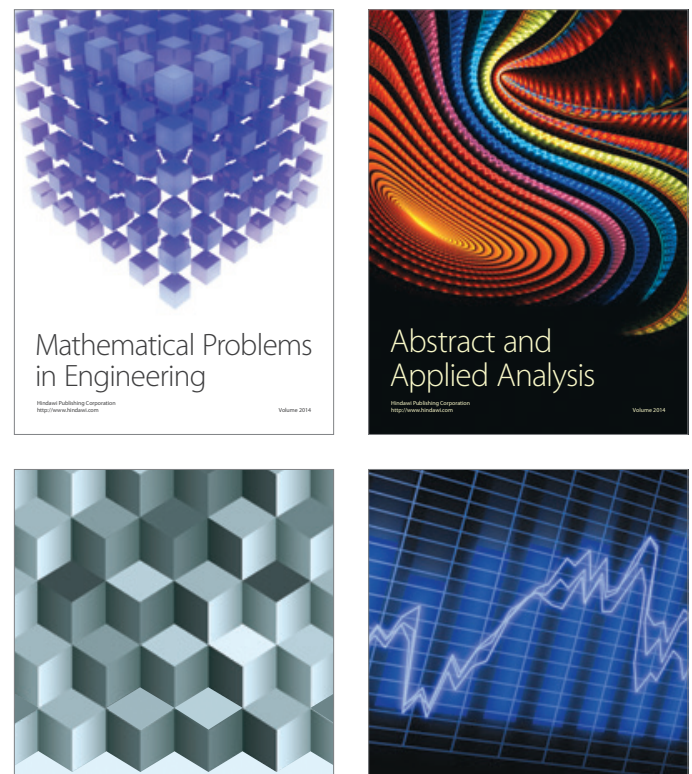

Journal of

Function Spaces

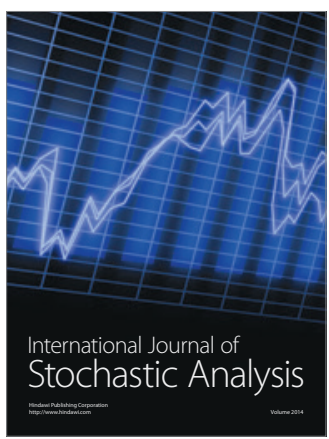

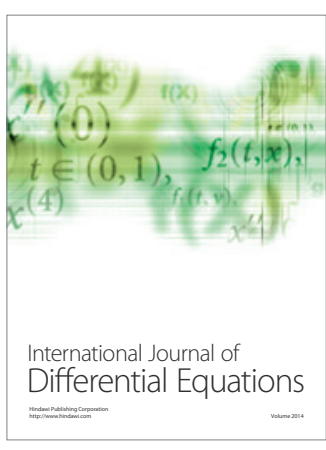
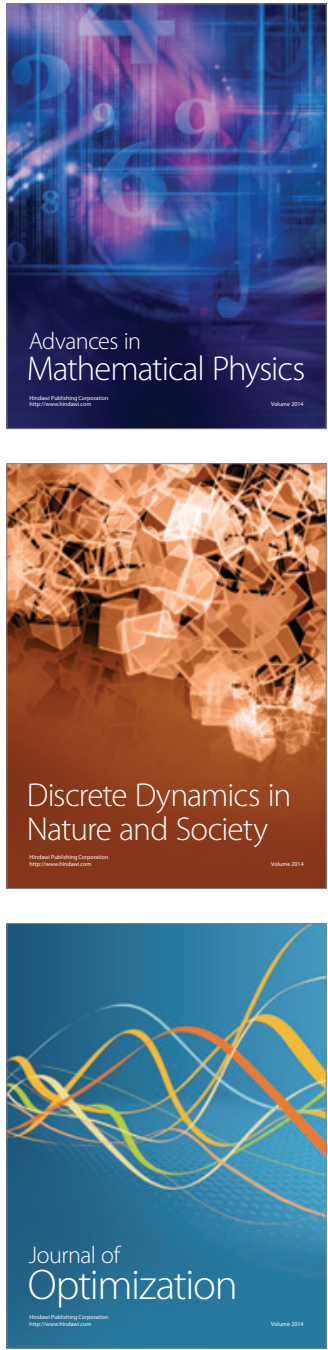